\title{
The Evolution of Magnetic Fields in Galaxy Clusters
}

\author{
Matthias Bartelmann, Klaus Dolag \\ Max-Planck-Institut für Astrophysik, P.O. Box 1317, D-85741 \\ Garching, Germany \\ Harald Lesch \\ Universitätssternwarte München, Scheinerstr. 1, D-81679 München, \\ Germany
}

\begin{abstract}
.
Cosmological simulations of magnetic fields in galaxy clusters show that remarkable agreement between simulations and observations of Faraday rotation and radio haloes can be achieved assuming that seed fields of $\sim 10^{-9} \mathrm{G}$ were present at redshifts $\sim 15-20$. The structure of the seed field is irrelevant for the final intracluster field. On average, the field grows exponentially with decreasing cluster redshift, but merger events cause steep transient increases in the field strength. Typical field-reversal scales are of order $50 \mathrm{~h}^{-1} \mathrm{kpc}$. In most cases, the intracluster fields are dynamically unimportant. Assuming secondary electron models, the average structure of cluster radio haloes can naturally be reproduced.
\end{abstract}

\section{Introduction}

Magnetised plasmas are optically birefringent. The orientation of polarised light traversing a magnetised plasma is therefore changed; this effect is called Faraday rotation. Being proportional to the square of the wavelength, it can be detected by observations of polarised radio sources in two or more frequency bands. Numerous such observations show that clusters contain magnetic fields which are smooth, i.e. ordered on scales comparable to the cluster scale (e.g. Vallee, MacLeod \& Broten 1986, 1987; Vallee 1990; Dreher et al. 1987; Kronberg 1987; Kim et al. 1990; Kim, Kronberg \& Tribble 1991; Clarke, Kronberg \& Böhringer 2001 to name only a few). Micro-Gauss field strengths are routinely inferred. Figure 1. illustrates data from Clarke et al. (2001). The Faraday rotation measure seen in polarised radio sources is plotted against their projected separation from the nearest Abell cluster. Evidently, the rms rotation measure increases substantially towards cluster centres, while it is compatible with zero at clustercentric distances beyond $\sim 1 h^{-1} \mathrm{Mpc}$.

This demonstrates that at least part of the Faraday rotation measured in sources behind galaxy clusters is due to intracluster magnetic fields rather than magnetic fields which are intrinsic to the sources themselves.

The Faraday rotation measure is proportional to the integral along the lineof-sight over the parallel component of the magnetic field, times the electron 


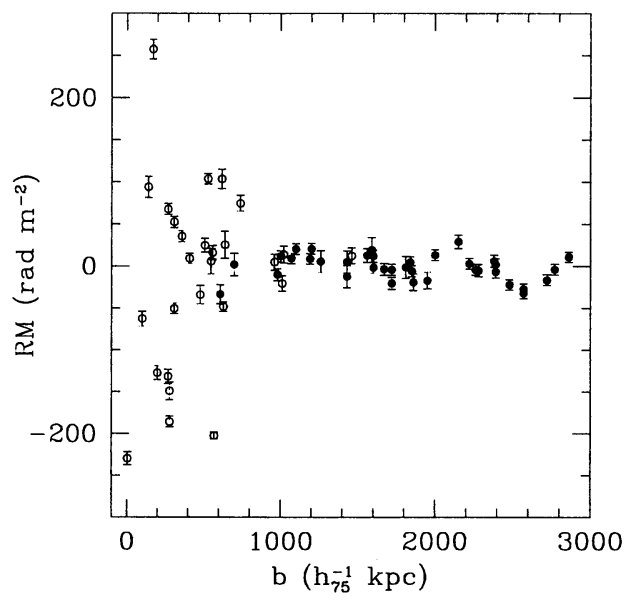

Figure 1. Data by Clarke et al. (2001) showing the Faraday rotation measure in radio sources against their projected distance from the nearest Abell cluster. While the rms rotation measure is compatible with zero beyond $1 \mathrm{Mpc}$, it increases substantially towards smaller clustercentric distances. This demonstrates the existence of smooth intracluster magnetic fields.

density. The same rotation measure can therefore be caused by strong fields which often change their direction, thus causing substantial cancellation, or weak fields which are almost homogeneous across a cluster. It is thus far from unique to infer magnetic field strengths from Faraday-rotation measures. Typically, fields with strengths of order $\mu \mathrm{G}$ are inferred, assuming simple magnetic-field geometries and typical electron densities derived from cluster $\mathrm{X}$-ray emission.

Another manifestation of intracluster magnetic fields is provided by radioand hard X-ray emission. Radio emission is caused by relativistic electrons gyrating in the intracluster field. The same population of relativistic electrons must Compton-upscatter photons of the cosmic microwave background into the hard X-ray regime. From the absence, or the low intensity, of this non-thermal, hard X-ray emission, lower limits can be inferred on the intracluster magnetic fields, because if the relativistic electron population is poor, the magnetic field has to be strong in order to explain the cluster radio haloes. These lower limits are typically also of micro-Gauss order (e.g. Bazzano 1990; Fusco-Femiano et al. 1999; Bagchi et al. 1998; Henriksen 1999).

This outlines the motivation for our numerical work summarised below. We wish to understand how magnetic fields evolve in galaxy clusters, how they act back on their host clusters themselves, how the initial conditions need to be set up so that observables like rotation measures and radio haloes can be reproduced, and how the magnetic fields are structured inside clusters.

\section{Numerical Technique}

For these simulations, we use the GrapeMSPH code (Dolag, Bartelmann \& Lesch 1999), developed from M. Steinmetz' (1996) GrapeSPH code. The code uses the Grape hardware (Ito et al. 1993) to trace dark-matter particles according to the softened force law

$$
\frac{\mathrm{d} \vec{v}_{a}}{\mathrm{~d} t}=-\sum_{i} \frac{m_{i}\left(\vec{r}_{a}-\vec{r}_{i}\right)}{\left(\left|\vec{r}_{a}-\vec{r}_{i}\right|^{2}+\epsilon_{a}^{2}\right)^{3 / 2}}
$$


where $a$ is the index of the particle under consideration, $\vec{r}_{a}$ and $\vec{v}_{a}$ are its position and velocity, and $\epsilon_{a}$ is its softening length. The summation extends over all other particles at positions $\vec{r}_{i}$ with masses $m_{i}$.

The dynamics of the gas is treated in the SPH approximation (Lucy 1977; Monaghan 1992). Particles are replaced by extended, soft spheres whose density is described by a kernel function $W$ with variable width $h$. Any physical quantity $A$ at position $\vec{r}$ is approximated as

$$
\langle A(\vec{r})\rangle \approx \sum_{i} m_{i} \frac{A\left(\vec{r}_{i}\right)}{\rho\left(\vec{r}_{i}\right)} W\left(\vec{r}-\vec{r}_{i}, h\right),
$$

where the sum extends over all particles which come sufficiently close to $\vec{r}$. The code combines the advantages of SPH, which is a fast method with adaptive resolution, with those of the Grape hardware, which provides supercomputer performance on low-end hardware. In our case, the host workstation is a twoprocessor SUN Ultra-Sparc 2300 equipped with five Grape-3Af boards.

The equation of motion for the gas particles can symbolically be written

$$
\begin{aligned}
\frac{\mathrm{d} \vec{v}_{a}}{\mathrm{~d} t} & =\text { pressure gradient }+ \text { viscous stresses }+ \text { magnetic force } \\
& + \text { gravity }+\Omega_{\Lambda} .
\end{aligned}
$$

In particular, the back-reaction of the magnetic field on the gas flow through the Lorentz force is included in the calculation. The term $\Omega_{\Lambda}$ symbolises the additional acceleration in presence of the cosmological constant. In addition to the momentum conservation expressed by Eq. (3), the energy equation is solved. The gas is treated as an ideal gas with an adiabatic index of 5/3. Cooling and heating are foreseen in the code, but have so far been used for testing purposes only.

We assume that the conductivity of the intracluster plasma is infinite, so that the induction equation of ideal magnetohydrodynamics can be used,

$$
\frac{\partial \vec{B}}{\partial t}=\nabla \times(\vec{v} \times \vec{B}) .
$$

Equation (4) and the inclusion of the Lorentz force in Eq. (3) constitute our main complements to the original GrapeSPH code.

The numerical viscosity is chosen such as to minimise viscous angular momentum transport in particle encounters (Monaghan \& Gingold 1983; Balsara 1995; Steinmetz 1996). The viscosity vanishes exactly in pure shear flows. Finally, all SPH expressions are symmetrised against particle exchange.

\section{Simulations}

We tested the code extensively, as described in detail in Dolag et al. (1999). Specifically, the code succeeds in computing spherical collapse models, and in solving shock tube problems (Brio \& Wu 1988). Although the code does not by construction conserve $\nabla \cdot \vec{B}$, it turns out in our simulations that $\nabla \cdot \vec{B}$ is always 
many orders of magnitude smaller than $|\vec{B}|$ divided by the correlation length of $\vec{B}$.

We perform simulations in two different cosmological models, SCDM with $\Omega_{0}=1$ and $\Omega_{\Lambda}=0$, and $\Lambda \mathrm{CDM}$ with $\Omega_{0}=0.3$ and $\Omega_{\Lambda}=0.7$. In both cases, we initialise dark matter distributions from the COBE-normalised CDM power spectrum. Galaxy clusters are surrounded a layer of more massive particles in order to mimic the gravitational tidal field exerted by neighbouring large-scale structure. The total particle number is limited by the Grape architecture, but our simulated clusters are generally resolved by $\geq 10^{4}$ particles.

Initial conditions are set up such that the average magnetic field strength at high redshifts (15 to 20 depending on cosmology) are of nano-Gauss order. In lack of any detailed prediction of the magnetic field structure at such redshifts, we use two vastly different assumptions. The field is either assumed to be homogeneous across the entire simulation volume, or chaotic, in which case its amplitude is drawn from a pre-defined power spectrum, and its direction is random, subject only to the condition that $\nabla \cdot \vec{B}=0$. Figure 2 shows one evolutionary stage of one of our cluster simulations as an example.

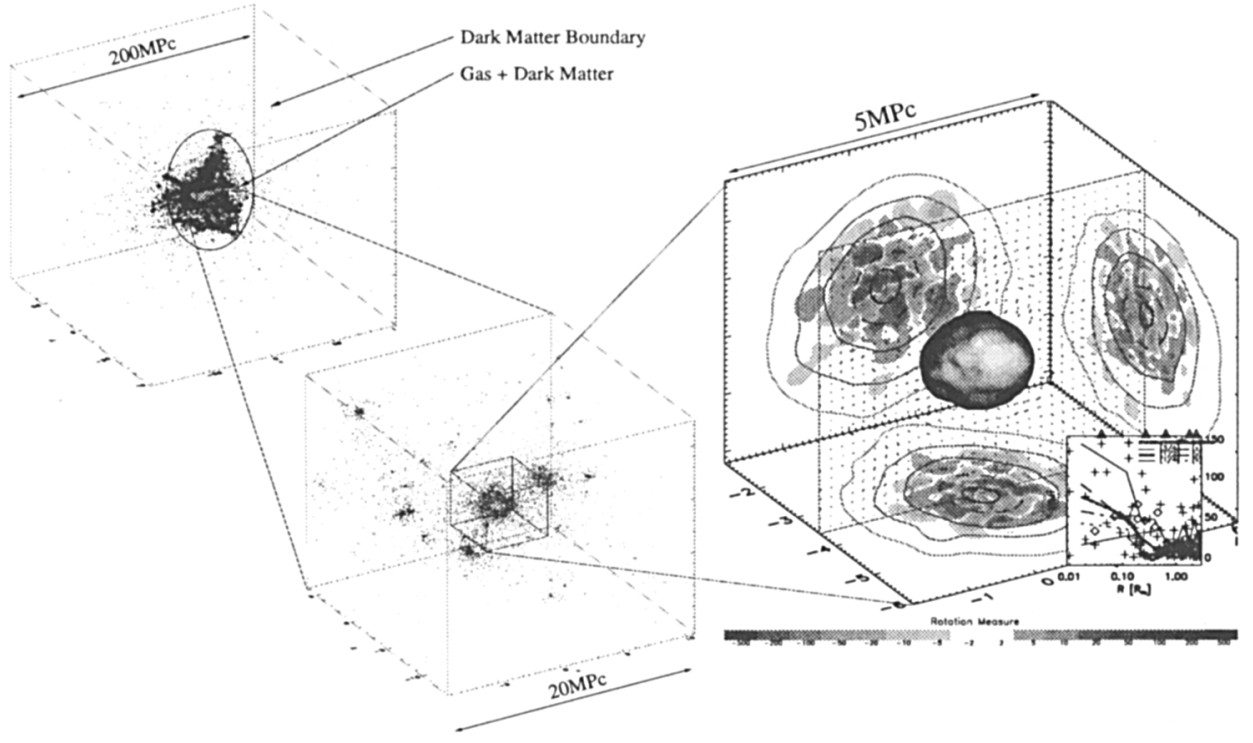

Figure 2. One evolutionary stage of one of our cluster simulations. Three boxes are shown which zoom into the cluster region from left to right. The right-most box has a side length of $5 h^{-1} \mathrm{Mpc}$. It shows a grey iso-density surface of the intracluster gas distribution in the centre; the grey contours on the box sides are isodensity curves of the intracluster gas. The coloured patches on the box sides show the Faraday rotation measure, with values ranging within $\pm 500 \mathrm{rad} \mathrm{m}^{-2}$. Red patches have negative, blue patches positive rotation measure. The purple frame in the middle of the box shows a slice through the intracluster magnetic field, with the arrows indicating field strength and orientation. 
The Faraday rotation measure created by the intracluster magnetic field is show as the coloured patches projected onto the box sides. The rotation measures range within $\pm 500 \mathrm{rad} \mathrm{m}^{-2}$, increasing from red to blue. Obviously, the rotation measures reach values typical for the observations, they cover a substantial portion of the cluster's cross section, and they show structure on scales of order $\sim 100 h^{-1} \mathrm{kpc}$. The figure also shows how the cluster is embedded into the surrounding large-scale structure, and it displays the strength and orientation of the magnetic field in a slice cut through the cluster core.

\section{Results}

\subsection{Field Structure and Amplification}

Starting from nano-Gauss fields at redshifts between 15 and 20, intracluster fields reach micro-Gauss strength in and near cluster centres at low redshift. Such field amplifications exceed expectations from simple spherical collapse models of a gas cloud with frozen-in magnetic field by about an order of magnitude. This becomes possible due to shear flows in the intracluster gas, which stretch, bend and entangle magnetic field lines and thereby increase the magnetic field strength. The left panel in Fig. 3 shows that the magnetic field strength, averaged within the central regions of many clusters in our simulated sample, increases approximately exponentially with decreasing redshift, $\langle|\vec{B}|\rangle \propto 10^{-2 z}$ (Dolag et al. 1999; Dolag, Bartelmann \& Lesch 2001).
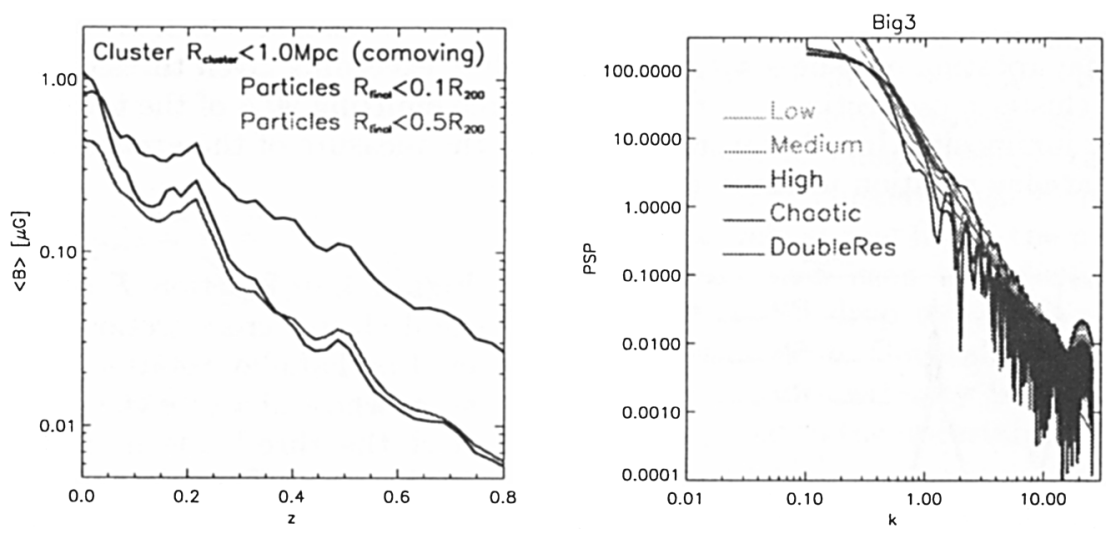

Figure 3. Left panel: Growth with redshift of the magnetic field strength averaged within many of our simulated clusters. On average, the field strength increases exponentially with decreasing redshift, approximately $\propto 10^{-2 z}$. - Right panel: Power spectra for the modulus of the intracluster magnetic field strength for various initial field set-ups. Above the Nyquist frequency of the simulation box, the power spectra are fairly steep power laws with exponents between -2.5 and -3 .

We find that the initial field structure is entirely irrelevant for the final field structure after cluster collapse. Simulations starting from the homogeneous 
magnetic field set-up lead to final magnetic fields which are statistically identical to those obtained from chaotic initial fields. This shows that the final structure of intracluster fields is completely determined by the cluster collapse, in the course of which any information on the initial field structure is erased.

The autocorrelation function of the magnetic field reveals typical fieldreversal scales of order $50 h^{-1} \mathrm{kpc}$. The right panel in Fig. 3 shows the power spectrum of the modulus of the final magnetic field strength for various initial conditions. The power spectrum resembles a power law for values of $k$ sufficiently larger than the Nyquist frequency of the box, quite independent of the initial field set-up. The power-law is fairly steep, with exponents ranging within -2.5 to -3 . This is steeper than expected for Kolmogorov turbulence, but compatible with two-dimensional magnetohydrodynamic turbulence. It cannot be excluded at this stage that the numerical viscosity of the SPH code contributes to steepening the magnetic-field power spectrum.

\subsection{Faraday Rotation}

Simulations in both cosmologies reproduce the statistics of observed Faradayrotation measures very well. On the whole, the amplitudes and coherence lengths of the simulated rotation-measure maps, and their projected radial profiles, agree excellently with the available data. As noticed before, the initial field configuration is irrelevant in that respect. Setting up fields with nano-Gauss strength at redshifts $15-20$ is sufficient.

Merger events in the cluster history are prominently reflected in the spatial distribution of the Faraday rotation measure. To illustrate this point, we show in Fig. 4.3. the fraction $\mathcal{F}$ of the total cluster cross section which is covered by Faraday rotation measures with absolute values exceeding given thresholds. The total cluster cross section is defined as the area emitting $90 \%$ of the total cluster $\mathrm{X}$-ray luminosity. In other words, we study the measure of the excursion set of the Faraday rotation measure map.

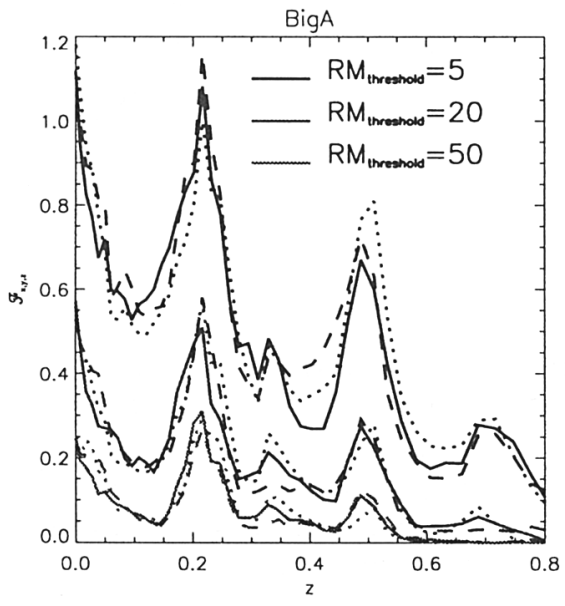

Figure 4. Fraction $\mathcal{F}$ of the total cluster cross section covered by Faraday rotation measures whose absolute values exceed the thresholds indicated in the plot. The total cluster cross section is defined to be the area emitting $90 \%$ of the total cluster luminosity. Irrespective of the threshold, the curves show steep, transient increases of $\mathcal{F}$ on top of a more gentle increase. The maxima coincide with the core passages following merger events.

Figure 4.2. shows a steady increase in $\mathcal{F}$ with decreasing redshift, underlying steep, transient increases which are independent of the threshold of the excursion 
set. Correlating these increases with the cluster evolution shows that $\mathcal{F}$ increases during merger events, reaching its maximum during core passage of the merged substructure, and decreasing thereafter as the cluster relaxes. Merger events therefore leave a pronounced imprint on the appearance of a cluster in Faradayrotation measurements.

Observationally, the rms rotation measure observed in galaxy clusters is closely correlated with their X-ray surface brightness. While this correlation contradicts simple analytic models for the intracluster magnetic fields, it is naturally reproduced in our simulated clusters (Dolag et al. 2001).

\subsection{Dynamics}

The intracluster magnetic fields provide pressure support adding to the thermal gas pressure. Hydrostatic equilibrium with the cluster potential wells can therefore be achieved with lower gas temperatures. However, the fields in our simulated clusters are typically so weak that the temperature reduction is of order a few per cent only. Although it thereby increases the scatter in the masstemperature relation to some degree, this effect is generally unimportant (Dolag, Evrard \& Bartelmann 2001).

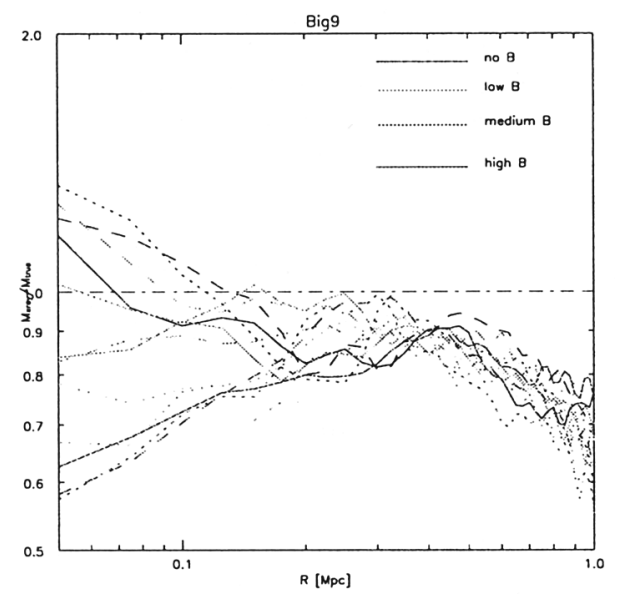

Figure 5. Masses inferred from $\beta$ models fit to magnetised clusters, divided by the true cluster masses, as a function of cluster-centric radius. For clusters with comparatively strong fields, the inferred mass near the cluster centres can drop to $\sim 60 \%$ of its true value. Generally, however, the uncertainty is small compared to the uncertainty in the $\beta$ model.

Almost everywhere in the simulated clusters, and almost always during their evolution, the magnetic fields are dynamically unimportant compared to the uncertainties in the standard $\beta$ model commonly used to infer cluster masses under the assumption of hydrostatic equilibrium. Lower cluster temperatures cause $\beta$-model mass estimates to be biased low. Figure 4.3 . shows that cluster masses inferred from $\beta$ models to the X-ray emission of magnetised galaxy clusters can be as low as $\sim 60 \%$ of the true cluster masses, but only in cluster cores and if the fields are comparatively strong. However, masses can substantially be underestimated in highly substructured or merging clusters (Dolag \& Schindler 2000). 


\subsection{Radio Haloes}

A natural consequence of intracluster magnetic fields is the development of smooth radio emission on cluster scales, so-called cluster radio haloes. Relativistic electrons in the intracluster plasma gyrate in the magnetic field and emit synchrotron radiation. Various models exist for the origin of the relativistic electron population. They can be primarily accelerated, but it is more likely that they from protons which are accelerated to relativistic energies in intracluster shocks. These protons decay into relativistic pions and electrons. Assuming such a secondary electron model, and putting between $5 \%$ and $15 \%$ of the thermal energy content into relativistic protons, our simulations can straightforwardly reproduce the radio halo of the Coma cluster (see Fig. 4.4.).

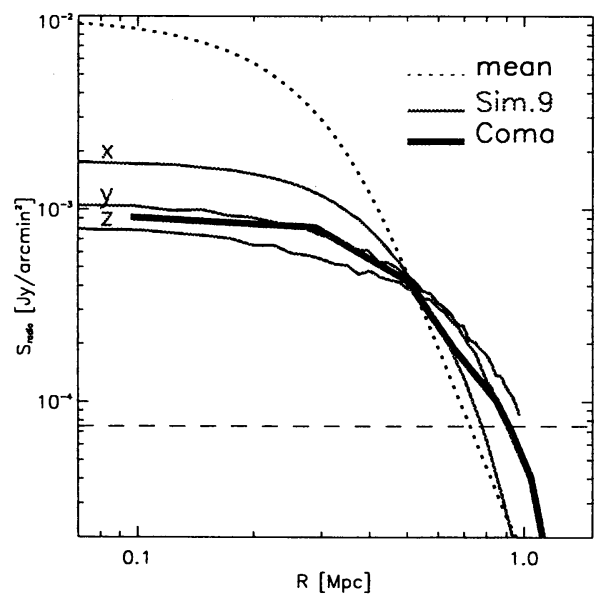

Figure 6. Radial profiles of the radio surface brightness for the Coma cluster (blue curve) and one of our simulated clusters assuming a secondary electron model (red curves). While this particular simulation reproduces Coma's radio-halo profile very well, the simulated radio profiles are on average somewhat steeper.

The figure shows the radial profile of the radio surface brightness in one of our simulated clusters (red curves) and in the Coma cluster (blue curve). While Coma's radio-halo profile is well reproduced by one particular simulation, the mean profile averaged across our complete simulated cluster sample is somewhat steeper. However, this can easily be modified by assuming that different spatial distributions of the relativistic protons from which the secondary electrons originate.

Remarkably, though, the very steep correlation between the cluster radio power and the X-ray temperature is naturally and accurately reproduced by our simulations (Dolag \& Enßlin 2000).

\section{Summary and Discussion}

We have performed cosmological simulations of magnetised galaxy clusters in two CDM cosmogonies, SCDM and $\Lambda$ CDM. The Grape hardware allowed these simulations to be efficiently executed on low-end hardware. We developed our GrapeMSPH code (Dolag et al. 1999) by augmenting Steinmetz' (1996) Grape$S P H$ code with the induction equation of ideal magnetohydrodynamics, and the Lorentz force for the back-reaction of the field on the intracluster gas. The code successfully passed an extensive suite of test runs. 
Our cluster simulations lead to the following results:

- Micro-Gauss fields at low redshift are reached starting from nano-Gauss fields at redshifts 15-20, depending on the cosmological model. Such field amplifications exceed by about an order of magnitude expectations from magnetic flux conservation in the simple spherical collapse model. They are possible due to shear flows in the intracluster gas.

- The initial structure of the magnetic fields is entirely unimportant for the final structure. Simulations starting from homogeneous magnetic fields lead to final fields which are statistically identical to those obtained from chaotic initial fields. The process of cluster formation wipes out any information on the initial field configuration.

- On average over many independent cluster simulations, the magnetic field strength grows exponentially with decreasing redshift.

- The simulations reproduce the observed statistics of Faraday-rotation measures very well. Cluster merger events lead to steep and transient increases in the area of the cluster which is covered by substantial rotation measures. This area is maximised during core passage of the merged substructure.

- Almost everywhere in the simulated clusters, and almost always during their evolution, magnetic fields are dynamically unimportant. They cause some increased scatter in the relation between $\mathrm{X}$-ray temperature and mass. During merger events, however, cluster masses inferred from $\beta$ fits can be lower than the true masses by factors of two or more.

- Assuming a secondary model for the production of relativistic electrons, and putting $5 \%-15 \%$ of the thermal cluster energy into relativistic protons, our simulated clusters have radio haloes which agree well with observed haloes. In particular, the very steep correlation between radio power and $\mathrm{X}$-ray temperature is naturally reproduced.

On the whole, it seems that our magnetohydrodynamic, cosmological cluster simulations capture many features displayed by observed galaxy clusters. However, the resolution of the simulations needs to be increased before detailed and reliable predictions can be made on the small-scale structure of intracluster magnetic fields and on the structure and emissivity of radio haloes. At a later stage, cooling of the intracluster gas has to be included, because it is expected to change substantially the structure and dynamical importance of magnetic fields in cluster cores.

Acknowledgments. We thank the organisers of IAU 208 for the very interesting and inspiring meeting. MB wishes to thank for generous financial support.

\section{References}

Bagchi, J., Pislar, V., Lima Neto, G.B. 1998, MNRAS 296, L23 
Balsara, D.S. 1995, J. Comput. Phys. 121, 357

Bazzano, A., Fusco-Femiano, R., Ubertini, P., Perotti, F. et al. 1990, ApJ 362, L51

Brio, M., Wu, C.C. 1988, J. Comput. Phys. 75, 400

Clarke, T.E., Kronberg, P.P., Böhringer, H. 2001, ApJ 547, L111

Dolag, K., Bartelmann, M., Lesch, H. 1999, A\&A 348, 351

Dolag, K., Bartelmann, M., Lesch, H. 2001, in preparation

Dolag, K., Enßlin, T. 2000, A\&A 362, 151

Dolag, K., Evrard, A.E., Bartelmann, M. 2001, A\&A 369, 36

Dolag, K., Schindler, S. 2000, A\&A 364, 491

Dolag, K., Schindler, S., Govoni, F., Feretti, L. 2001, A\&A in press; preprint astro-ph/0108485

Dreher, J.W., Carilli, C.L., Perley R.A. 1987, ApJ 316, 611

Fusco-Femiano, R., dal Fiume, D., Feretti, L., Giovannini, G., Grandi, P., Matt, G., Molendi, S., Santangelo, A. 1999, ApJ 513, 21

Henriksen, M., 1999, ApJ 511, 666

Ito, T., Makino, J., Fukushige, T., Ebisuzaki, T., Okamura, S.K., Sugimoto, D. 1993, PASJ 45, 339

Kim, K.T., Kronberg, P.P., Dewdney, P.E., Landecker, T.L. 1990, ApJ 355, 29

Kim, K.T., Kronberg, P.P., Tribble, P.C. 1991, ApJ 379, 80

Kronberg, P.P. 1987, in: Interstellar magnetic fields: Observations and Theory. Berlin \& New York: Springer-Verlag

Lucy, L. 1977, AJ 82, 1013

Monaghan, J.J. 1992, ARA\&A 30, 543

Monaghan, J.J., Gingold, R.A. 1983, J. Comput. Phys. 52, 374

Steinmetz, M. 1996, MNRAS 278, 1005

Vallee, J.P., MacLeod, J.M., Broten, N.W. 1986, A\&A 156, 386

Vallee, J.P., MacLeod, J.M., Broten, N.W. 1987, ApL 25, 181

Vallee, J.P. 1990, AJ 99, 459 